\title{
Tripoli dans les réseaux d'échanges intercontinentaux à la fin du Moyen Âge
}

\author{
Dominique VAlÉRIAN*
}

La particularité de Tripoli au Moyen Âge est d'être dans une position à la fois centrale et marginale dans l'organisation de l'espace politique et économique des pays d'Islam et de la Méditerranée. Par sa position géographique, c'est une évidence, elle est à la charnière des deux bassins oriental et occidental de la Méditerranée, mais aussi à la frontière de l'Orient et de l'Occident musulman. Très tôt les géographes arabes, en dépit d'un certain flou parfois, font en effet commencer le Maghreb dâns cette région, souvent à Syrte. Cela recoupe une division politique qui s affirme très vite, entre les provinces relevant du gouverneur d'Égypte, jusqu’à la Cyrénaïque, et celles, reconnaissant l'autorité du gouverneur de Kairouan, intégrées dans la province d'Ifrīqiya, jusqu'à la Tripolitaine ${ }^{1}$. Elle est donc dans une position marginale par rapport à ces deux grands ensembles, en dépit d'une intégration régionale opérée dans le cadre de l'empire islamique, puis d'une Méditerranée de plus en plus unifiée et structurée par dés réseaux d'échanges étendus, ce qui la place dans une position intermédiaire sur différents réseaux en relation avec le Maghreb et l'Orient, l'Afrique sub-saharienne et la Méditerranée (carte en annexe).

Cette situation particulière, aux marges de l'Ifrīqiya mais aussi dans une position stratégique pour le contrôle de la navigation en Méditerranée centrale et de la route terrestre entre le Maghreb et l'Orient, explique qu'elle fut l'objet d'âpres compétitions : les maîtres du Caire, à plusieurs reprises, tentent de la faire passer sous leur domination, par exemple sous le règne de Saladin à la faveur des troubles causés par les Banū Ghāniya - Qarāqūš la

\footnotetext{
* Professeur d'histoire médiévale à l'Université Lumière-Lyon 2 / CIHAM.

1. Jusqu’à Labda à l'époque Aghlabide (M. TALBI, L'Émirat aghlabide, histoire politique, 800909, Paris, 1966, p. 125, et carte p. 127), avec quelques visées égyptiennes mais toujours ponctuelles. Cf. J. Thiry, Le Sahara libyen dans l'Afrique du Nord médiévale, Louvain, 1995, p. 76.
} 
gouverna alors pendant un temps au nom du sultan ayyūbide. Les chrétiens s'y intéressent également, et la ville est occupée en 1145 par le roi Normand Roger II, puis en 1354 ou 1355 par l'amiral génois Filippo Doria, qui la tient pendant six mois ${ }^{2}$. Cette position permet surtout à Tripoli de se rendre indépendante à plusieurs reprises, son éloignement par rapport à la capitale rendant son contrôle plus difficile en période de défaillance du pouvoir : ainsi au début du XI ${ }^{\mathrm{e}}$ siècle les Banū Khazrūn, une puissante famille de la tribu Maghrāwa, établissent une seigneurie qui s'appuie sur un conseil de notables $(s h \bar{u} r \bar{a})$ et reconnaissent une tutelle assez lâche des califes fatimides du Caire et parfois de leurs voisins zirides de Kairouan - ce qui ne les empêche pas de se rapprocher un temps des califes omeyyades de Cordoue ${ }^{3}$. Après l'arrivée des Hilaliens en Tripolitaine au milieu du siècle, ils conservent le pouvoir sur la ville - peut-être en passant des accords avec les Arabes.

À nouveau à l'époque hafside Tripoli parvient à se rendre indépendante des sultans de Tunis, à la faveur des difficultés internes de la dynastie et en s'appuyant sur les tribus arabes installées dans la région depuis le $\mathrm{XI}^{\mathrm{e}}$ siècle. $\mathrm{Au}$ milieu du XIv siècle, alors que le pouvoir hafside traverse une très grave crise, elle est intégrée aux domaines des Banū Makkī, une tribu arabe qui, à partir de Gabès, se taille un domaine largement orienté vers la mer et le contrôle des ports, avec la possession de Sfax, de Djerba et des îles Kerkennah. Mais c'est surtout dans les dernières décennies du XIv siècle que la ville parvient à se rendre totalement indépendante, sous la conduite d'une famille de notables appartenant sans doute à l'élite marchande du port, les Banū Thābit. Elle prend une première fois le pouvoir en 1324, mais doit l'abandonner après la prise de la ville par les Génois puis son passage sous le contrôle des Banū Makkī. Thābit doit alors fuir Tripoli et se rend en Égypte. Ce choix - les princes déchus désireux de reconstituer leurs forces pour une reconquête du pouvoir se réfugient plus volontiers dans les tribus de l'intérieur - est intéressant et peut laisser penser à l'existence de liens privilégiés, qui pourraient être de nature commerciale, avec l'Égypte. Quoi qu'il en soit, à Alexandrie les Banū Thābit s'adonnent au commerce et leur exil forcé leur permet de s'enrichir suffisamment pour être en mesure de financer leur retour au pouvoir en nolisant des

2. Iвn Khaldūn, Kitāb al-'Ibar, Beyrouth, 1959, vol. 6, p. 616 ; iD., Histoire des Berbères et des dynasties musulmanes de l'Afrique Septentrionale, W. MaC Guckin DE SLANe, trad., revue par P. Casanova, Paris 1956, vol. 3, p. 174 (1 ${ }^{\text {re }}$ éd. Alger, 1852-1856) ; E. Rossi, Storia di Tripoli e della Tripolitania dalla conquista araba al 1911, Rome, 1968, p. 90-91.

3. E. Rossi, Storia di Tripoli..., op. cit., p. 53-56. 
navires chrétiens et en louant les services d'Arabes de la région de Tripoli ${ }^{4}$. Ils parviennent ainsi en 1369 à se rendre maîtres de la ville en l'attaquant à la fois par terre et par mer, et la gouvernent nominalement au nom des Hafsides : ils lèvent pour eux-mêmes les taxes dans la région et versent (de plus ou moins bonne grâce) un tribut à Tunis. L'aide du roi de Sicile leur permet de se prémunir des attaques hafsides qui cherchent à rétablir leur pleine souveraineté à la fin du siècle ${ }^{5}$, et ce n'est qu'en 1400-1401 que le sultan de Tunis Abū Fāris parvient enfin à les chasser du pouvoir.

Cette capacité de Tripoli à rester longtemps autonome, fait relativement rare pour les villes de l'Islam médiéval, tient à des conjonctures particulières, mais aussi à un certain nombre de caractéristiques qui lui sont propres : outre l'éloignement par rapport aux centres du pouvoir en Ifrīqiya et en Égypte, elle a vu se développer une élite qui tire une partie de sa puissance des affaires commerciales du port, et peut bénéficier, pour résister aux efforts hafsides de reconquête, des ressources et des secours éventuels apportés par mer. Ses liens avec l'hinterland sont en effet assez particuliers : la présence dans le djebel Nafūsa de communautés ibadites, promptes à la révolte contre le pouvoir sunnite de Kairouan, mène dès l'époque aghlabide à un partage de fait du territoire, entre l'intérieur tenu par les ibadites et le littoral, avec la capitale provinciale Tripoli, qui reste sous le contrôle de Kairouan et permet d'assurer la sécurité de la route stratégique menant en Orient ${ }^{6}$. Après le milieu du $\mathrm{XI}^{\mathrm{e}}$ siècle, la mainmise sur les régions intérieures par les tribus arabes venues d'Égypte confirme cette partition politique. Des accords sont sans doute conclus, qui permettent à la ville de se fournir en produits alimentaires et surtout d'assurer la sécurité des marchands commerçant avec les régions sahariennes, notamment par l'intermédiaire de Zawilla et du Fezzan ${ }^{7}$.

L'importance du port s'accroît encore à partir du XI ${ }^{\mathrm{e}}-\mathrm{XII}^{\mathrm{e}}$ siècle, sous l'effet de deux évolutions contemporaines. D'une part l'instabilité chronique

4. Ibn Khaldūn, Kitāb al-'Tbar, op. cit., éd. VI, p. 616, trad. III, p. 174.

5. E. Rossi, Storia di Tripoli..., op. cit., p. 92. L'auteur cite un échange d'ambassades entre Tripoli et la cour de Sicile.

6. Iвn AL-Athīr, Al-Kämil fì al-tảìikh, A. A. AL-Q̄̄oì éd., Beyrouth, 1987, vol. 4, p. 188 ; Annales du Maghreb \& de l'Espagne, E. Fagnan trad., Alger, 1901, p. 176 ; M. TALBI, L'Émirat aghlabide..., op. cit., p. 158.

7. J. Thiry, Le Sahara libyen..., op. cit., notamment p. 199-203, 399 et suiv. ; E. SAVAGE, "Berbers and Blacks : Ibadi slave traffic in eighth-century North Africa ", The Journal of African History, 33/3 (1992), p. 351-368. L'auteur fait remonter le commerce ibadite dans cette région à la fin du viII ${ }^{\mathrm{e}}$ siècle. 
et l'insécurité des routes terrestres s'amplifient durablement à la suite de l'arrivée de tribus arabes nomades et des troubles consécutifs au conflit entre les Almohades et les Banū Ghāniya entre la fin du XII et le début du XIII ${ }^{e}$ siècle $^{8}$. La conséquence principale est le fort ralentissement du commerce terrestre entre le Maghreb et l'Égypte, ce dont témoignent les documents de la Geniza : alors que jusqu'au milieu du XI ${ }^{\mathrm{e}}$ siècle un voyage sur vingt se faisait par voie de terre, le ratio passe à un pour cinquante par la suite'. Dès lors, la route terrestre entre l'Orient et l'Occident musulmans reste, jusqu'à la fin du Moyen Âge, un axe difficile et dangereux, soumis à des accords toujours fragiles avec les tribus arabes. Concrètement, ces relations se limitent en grande partie au passage de la caravane du pèlerinage ${ }^{10}$, qui continue à emprunter la voie terrestre par la Tripolitaine et nécessite désormais une lourde organisation, notamment pour en assurer la protection $^{11}$. Les échanges commerciaux par cette voie semblent en revanche des plus limités, et les rares marchands ou voyageurs qui empruntent toujours cette route doivent compter avec les risques du brigandage ${ }^{12}$. Le fait qu'au

8. Jacques Thiry considère que ce sont les Banū Ghāniya qui sont les premiers responsables de la crise de la région, faisant remarquer qu'al-Idrīsĩ décrit des ports encore prospères. (J. Thiry, Le Sahara libyen..., op. cit., p. 236-278). Mais il est plus probable que les deux problèmes se conjuguent sur la longue durée.

9. S. D. Goitein " The unity of the Mediterranean world in the "Middle" Middle Ages ", Studia Islamica, 12 (1960), p. 36.

10. En 872/1468 le sultan de Tunis 'Uthmān écrit à celui du Caire Qāitbāy pour l'informer de la venue de la caravane du pèlerinage accompagnée d'un ambassadeur hafside muni de cadeaux pour le sultan mamelouk. G. S. Cousné, "Contribution à l'étude des relations diplomatiques entre les musulmans d'Occident et l'Égypte au $\mathrm{Xv}^{\mathrm{e}}$ siècle ", dans Mélanges Maspero. III : Orient islamique, Le Caire, 1935-1940, p. 205-206.

11. Une fatwa postérieure au règne du sultan mérinide Abū 'Inān (1348-58) raconte un événement survenu peu de temps auparavant : une caravane de pèlerins, forte de plus de 20000 hommes et chevaux, avait été laissée tranquille par les tribus trop faibles, mais fut attaquée par des tribus puissantes et dut payer, après les combats, une somme considérable pour s'échapper. Le mufti reconnaît alors que beaucoup de savants et de dévots n'ont pas accompli le pèlerinage, et que seuls ceux qui ne possèdent rien peuvent s'aventurer sur cette

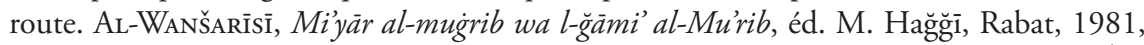
p. 441-442 ; analyse V. Lagardère, Histoire et société en Occident musulman au Moyen Âge. Analyse du Mì̀yār d'al-Wanšarìsī, Madrid, 1995, p. 38.

12. Au XIII' siècle al-'Abdarī, qui prend également la voie terrestre, ne décrit que ruines en Tripolitaine et Cyrénaïque (J. Thiry, Le Sahara libyen..., op. cit., p. 277, 304, 310). Il en est de même d'al-Tiğānī au début du xive siècle. M. BRETT, "Arabs, Berbers and Holy Men in Southern Ifriqiya, 650-750H/1250-1350 AD. ", Les Cahiers de Tunisie, 29 (1981), p. 548. L'auteur note cependant l'existence de marchés, notamment autour de zāwiyas fon- 
début du XIV siècle une ambassade mamelouke envoyée au Maroc profite du départ de la caravane du pèlerinage de retour des Lieux Saints pour traverser la Tripolitaine, comme trois ans plus tard l'ambassade mérinide envoyée au Caire ${ }^{13}$, laisse penser qu'en dehors de ce moment du pèlerinage les voyages étaient rares et dangereux ${ }^{14}$.

Cette évolution de la situation intérieure contribue à donner un peu plus d'importance au port de Tripoli, un des plus actifs de la région. Il profite de la seconde mutation majeure de cette période charnière, l'essor contemporain de la navigation et du commerce méditerranéen des puissances chrétiennes, notamment italiennes. À partir du XII ${ }^{\mathrm{e}}$ siècle en effet une grande partie du commerce maritime est prise en main par les flottes marchandes latines, alors que les acteurs du monde musulmans se limitent pour l'essentiel au commerce terrestre. Les liaisons entre l'Égypte et le Maghreb ne sont pas interrompues, mais obéissent désormais à d'autres logiques liées à des réseaux dont les pôles sont situés à Gênes, Venise ou plus tard Barcelone. Il n'est pas rare en particulier de trouver des exportations de produits orientaux comme les épices qui arrivent dans les ports maghrébins depuis Gênes ou Marseille, après un périple qui les fait transiter par l'Europe. Certains navires chrétiens continuent de relier Alexandrie à Tunis, mais ce commerce triangulaire rend cette route directe moins nécessaire et elle est sans doute moins fréquentée qu'au cours des $\mathrm{IX}^{\mathrm{e}}-\mathrm{XI}^{\mathrm{e}}$ siècles ${ }^{15}$. Tripoli reste une des destinations fréquente sdes navires chrétiens, ce qui contribue à sa prospérité et est sans doute une des raisons, nous l'avons vu, de sa capacité à demeurer indépendante

dées par des hommes pieux appartenant parfois aux tribus arabes et qui tentent d'apporter leur aide aux voyageurs par leur baraka (Ibid., p. 552).

13. M. Снароutot-REMADI, «Les relations entre l'Égypte et l'Ifrîqiya aux XIII et XIV siècles d'après les auteurs mamlûks ", dans Actes du premier congrès d'histoire et de civilisation du Maghreb. I, Tunis, 1979, p. 156-157 (Cahiers du CERES, Série histoire, 1). C’est également le cas d'Ibn Battūta, qui décrit une caravane accompagnée de cent cavaliers ou plus, escortée par un détachement d'archers qui permit de garder les nomades à l'écart ("Voyages et périples (Ribla). Présent à ceux qui aiment à réfléchir sur les curiosités des villes et les merveilles des voyages ", dans Voyageurs arabes, P. Charles-Dominique trad., Paris, 1995, p. 381).

14. Subhi Labib constate également une correspondance entre l'époque du pèlerinage et celle du commerce par voie terrestre entre le Maghreb et l'Égypte, les sultans mamelouks assurant la sécurité sur la route de Barca par des expéditions régulières. S. Y. LaBiB, Handelsgeschichte Ägyptens im Spätmittelalter (1171-1517), Wiesbaden, 1965, p. 99.

15. S. D. Goitein, " La Tunisie du XI ${ }^{\mathrm{e}}$ siècle à la lumière des documents de la Geniza du Caire ", dans Études d'orientalisme dédiées à la mémoire de Lévi-Provençal. II, Paris, 1962, p. 559-579. 
pendant de longues décennies. Toutefois, son activité dépend étroitement de son attractivité pour les marchands européens, et est donc soumise aux mutations de réseaux dont les pôles d'impulsion sont désormais situés sur la rive nord de la Méditerranée.

Le port apparait très tôt dans les sources latines : le plus ancien document génois faisant état d'un commerce avec le monde musulman, un tarif de douane, conservé dans un registre de copies de documents relatifs à l'archevêché de Gênes et compilé en 1143, cite les marchandises venant de Tripoli de Berbérie ${ }^{16}$. On trouve également le port dans le minutier du notaire génois Giovanni Scriba, le plus ancien conservé, daté du milieu du XII ${ }^{\mathrm{e}}$ siècle $^{17}$. En revanche, Tripoli ne figure pas dans la liste des ports ouverts aux Pisans dans le traité signé avec les Almohades en 1186. Pourtant jusqu’à la fin du XIV siècle, Tripoli reste relativement marginale dans les investissements des marchands latins, par rapport à Tunis et Alexandrie. Elle est surtout un point d'appui pour la navigation entre ces deux villes - ce qui peut d'ailleurs expliquer qu'elle apparaisse peu dans les contrats, les opérations réalisées dans les ports intermédiaires étant rarement prévues lors de la signature du contrat. Certains indices montrent cependant une activité marchande importante : dans le traité de 1234, entre Pise et les Hafsides, il est demandé que l'on y bâtisse un fondouk pour accueillir les marchands toscans ${ }^{18}$; et lors de la prise de la ville par les Génois en 1355, Ibn Khaldūn explique le succès de l'entreprise par la ruse des chrétiens venus sous couvert de navires de commerce, «sans que personne n'y fit attention, vu le grand mouvement commercial qui y régnait et la fréquence des arrivages et des départs ${ }^{19}$ ".

C'est peut-être à cette époque, au milieu du XIv ${ }^{e}$ siècle, que Tripoli commence à se détacher et à prendre une autonomie commerciale par rapport à

16. «Registro della Curia arcivescovile di Genova », L. T. Belgrano éd., Atti della Società Ligure di Storia Patria, 2 (1862), p. 9, 365. Elle figure aux côtés de Mahdia (Africa), Tunis, Bougie et Almeria.

17. D. Abulafia, "L'attività commerciale genovese nell'Africa normanna : la città di Tripoli ", dans Atti del Congresso internazionale di studi sulla Sicilia normanna, Palerme, 1974, p. 395-402 ; G. PIstarino, « Navi e mercanti a Tripoli e in Barbaria (Genova, secoli $\mathrm{XII}-\mathrm{XV})$ : aspetti storici e giuridici ", dans Historia económica y de las instituciones financieras en Europa. Trabajos en homenaje a Ferran Valls i Taberner, M. J. Peláez dir., Barcelone, 1989, p. 3397-3401.

18. Traités de paix et de commerce et documents divers concernant les relations des Chrétiens avec les Arabes de l'Afrique septentrionale au Moyen-Âge, L. DE Mas LATrie, éd., Paris, 1866, p. 34 (art. 15).

19. Iвn Khaldūn, Kitāb al-'Ibar, op. cit., op. cit., éd. VI, p. 616, trad. III, p. 173. 
Tunis et Alexandrie. Le traité signé à Tripoli en 1356, entre Venise et les Banū Makkī, couvre les terres allant de Sfax à Misrata et accorde une place majeure au port, où se trouvent le consul et le fondouk vénitiens. Par ailleurs, la correspondance diplomatique avec la République dans les années qui suivent montre un intérêt notable pour cette destination. Le traité proprement dit porte principalement sur l'exploitation du sel de Räs al-Makhbaz, dont l'exportation par les Vénitiens ne passait pas nécessairement par Tripoli, mais aussi sur les tarifs douaniers applicables dans le port ${ }^{20}$. Cet intérêt est en partie lié à une situation politique particulière, et à l'affaiblissement des Hafsides de Tunis, mais aussi à une évolution plus durable marquée notamment par la réautorisation par la papauté du commerce avec l'Égypte mamelouke au milieu du XIV siècle, qui redonne à Tripoli un rôle important sur la route d'Alexandrie.

La crise de la seconde moitié du XIv ${ }^{e}$ siècle, avec notamment les effets de la peste noire, n’a cependant pas permis un véritable décollage du commerce, et c'est surtout au $\mathrm{Xv}^{\mathrm{e}}$ siècle, lorsque reprend vraiment l'activité commerciale en Méditerranée, que Tripoli s'affirme comme un des ports les plus importants de la rive africaine. Le port abrite alors un arsenal, utilisé notamment pour construire des navires pour les corsaires ${ }^{21}$, et surtout attire de plus en plus les marchands chrétiens. Cette affirmation de la place de Tripoli, en dépit de sa réintégration dans les territoires contrôlés par Tunis, s'explique par l'intérêt nouveau qu'elle présente aux yeux des marchands latins, à la fois pour les produits qu'elle est susceptible de fournir et par la place qu'elle occupe dans les réseaux d'échanges qui se reconstruisent au Xve siècle.

Les produits que les marchands chrétiens viennent chercher à Tripoli sont en partie communs à ceux que l'on trouve dans d'autres régions du Maghreb, notamment les produits de l'élevage, comme les cuirs et les laines, très recherchés pour les industries européennes. Le traité de 1356 mentionne également l'huile et les dattes, mais il est difficile de savoir s'il s'agit de productions exportées depuis Tripoli ou depuis d'autres ports tenus alors par les Banū Makkī, entre autres, dans le Sahel tunisien. De même le sel, qui fait l'objet d'un développement important dans ce traité, est plutôt celui

20. Traités de paix et de commerce..., op. cit., p. 224.

21. En 1400 des informations parviennent à Majorque par un marchand présent à Collo à propos d'une aide de 20 à 25 navires de guerre que le sultan aurait promise au corsaire Pere Fuster, les galères étant construites à Collo, Bougie, Tunis, Djerba et Tripoli à raison de deux par arsenal, et les galiotes dans d'autres ports. M. D. López Pérez, La Corona de Aragón y el Magreb en el siglo XIV (1331-1410), Barcelone, 1995, p. 712-713. 
de Rās al-Makhbaz, et il n’est pas sûr qu'il transite par Tripoli, étant plutôt embarqué sur les plages proches des zones de production. Ce qui fait l'intérêt du port, et le distingue des villes plus occidentales, est le commerce des esclaves noirs, qui se développe à partir du $\mathrm{Xv}^{\mathrm{e}}$ siècle, surtout dans sa seconde moitié, dans les Monts de Barca et, dans une moindre mesure il est vrai, à Tripoli. Ces esclaves étaient apportés par caravane depuis la région du Kanem-Bornou, autour du Lac Tchad, soit au sud de la Tripolitaine et de la Cyrénaïque, auxquelles la reliaient des routes anciennes passant par le Fezzan et Zawilla. Au début du Xve siècle le Vénitien Emmanuel Piloti parle ainsi de l'arrivée chaque année en Égypte de mille à deux mille jeunes esclaves noirs du "pays du roy de Tune et de la Barbarie, Tripoli $~^{22}$. Certains sont d'ailleurs apportés par les Vénitiens à bord des galées du Trafego qui assurent la liaison entre Tunis et Alexandrie : vers 1480 par exemple, un marchand vénitien apporte 188 noirs et d'autres femmes esclaves noires de Tripoli de Libye à Alexandrie ${ }^{23}$. Mais ils sont surtout exportés à destination des marchés européens, principalement par des marchands catalans basés en Sicile, d'où ces derniers redistribuent leur marchandise dans l'ensemble des territoires de la couronne d'Aragon et au-delà. Ce mouvement commercial prend une ampleur particulière entre les années 1410-1420 et les années 1470 , soit entre le moment où l'avancée ottomane compromet les sources traditionnelles d'approvisionnement en esclaves de Romanie, et l'ouverture des routes atlantiques de la traite africaine par les Portugais.

Lexportation de main d'œuvre servile depuis un port musulman en direction du monde chrétien pose cependant un problème, dans la mesure où les esclaves sont considérés comme un produit stratégique, dont la vente à des ennemis de la foi est en théorie interdite, dans un sens comme dans l'autre. Le fait que ce commerce se développe précisément dans cette région où la souveraineté de Tunis (ou du Caire pour les Monts de Barca) est moins affirmée n'est peut-être pas un hasard, et constitue en tout cas une spécificité qui sans doute explique, en partie au moins, l'essor de l'activité du port de Tripoli au Xve siècle.

L'autre raison de l'essor de l'activité de Tripoli au Xve siècle tient à la modification des réseaux d'échanges en Méditerranée. L'importance du marché égyptien, de nouveau ouvert aux chrétiens, comme les liaisons avec les ports

22. E. Piloti, Traité d'Emmanuel Piloti sur le passage en Terre Sainte (1420), P.-H. DopP éd., Louvain/Paris, 1958, p. 135.

23. E. Ashtor, "The Venetian supremacy in Levantine trade : monopoly or pre-colonialism ? ", The Journal of European Economic History, 3/1 (1974), p. 29. 
de l'Atlantique, dans les Flandres et en Angleterre, poussent à intensifier, plus encore qu'auparavant, les liaisons de direction est-ouest le long des côtes africaines. Le système vénitien des mude montre bien cette évolution, avec la création en 1460 de la muda du Trafego, qui reliait Tunis à l'Égypte en passant par Djerba et Tripoli ${ }^{24}$. Les Florentins font de même, avec moins de succès il est vrai, en mettant en place en 1447 une ligne orientale de galées, qui passait par Naples et la Sicile pour gagner Tunis et Tripoli, prolongée parfois jusqu’à Alexandrie ${ }^{25}$. C'est également à cette époque que l'on constate que les navires qui partent de Sicile vers l'est ne passent plus nécessairement par l'escale tunisoise, et font voile directement vers Tripoli, avant de continuer vers l'est en longeant la côte et notamment les Monts de $\mathrm{Barca}^{26}$. Enfin on constate que de plus en plus souvent des navires suivent des itinéraires complexes en reliant des ports des deux bassins de la Méditerranée, sans nécessairement repasser par le port d'attache, ce qui était jusque-là la règle générale : en 1443 à Valence, les patrons de deux galées marchandes établissent ainsi un contrat de navigation de conserve, qui prévoit l'itinéraire suivant : elles doivent quitter Valence en février, passer par Barcelone (cinq jours d'escale), rejoindre Palerme (six jours...), Tunis, d'où une des deux galées ira à Majorque charger des marchandises puis rejoindra Tunis par Alger (trois jours...), Bougie (deux jours...). De Tunis, les deux navires repartiront pour Tripoli (quatre jours...), Rhodes, (trois jours...), Alexandrie (quarante-cinq...), avant de revenir par Rhodes $^{27}$. Dans cette nouvelle configuration des réseaux de navigation européens, Tripoli n'est plus un angle mort entre les deux marchés porteurs de l'Ifrīqiya et de l'Égypte, mais au contraire sur le passage des grandes routes de navigation de direction est-ouest.

Tripoli devient donc au $\mathrm{XV}^{\mathrm{e}}$ siècle un pivot dans des réseaux associant des axes est-ouest et nord-sud essentiels pour les marchands européens. La place que la ville occupe dans les derniers siècles du Moyen Âge tient d'abord à sa situation dans l'espace musulman, notamment comme un des débouchés du commerce des esclaves d'Afrique sub-saharienne, mais aussi dans les réseaux

24. B. Doumerc, Venise et l'émirat hafside de Tunis (1231-1535), Paris, 1999, p. 105.

25. Ibid., p. 73-75. Cette ligne florentine n'a cependant ni la régularité, ni le succès de celle organisée par Venise, et après 1466 elle ne fonctionne plus guère (à part en 1478).

26. H. Bresc, Un Monde méditerranéen : économie et société en Sicile, 1300-1450, Rome, 1986 , p. 335.

27. E. Cruselles Gómez « La organización del transporte marítimo en la Valencia de la primera mitad del siglo xv », Anuario de Estudios Medievales, 24 (1994), p. 175-176. 
d'échanges maritimes organisés par les marchands européens. Pendant longtemps cependant elle occupe une place relativement secondaire et sert principalement d'escale sur la route reliant les deux pôles ifrīqiyen et égyptien. Ce n'est qu'au milieu du XIV siècle, et surtout au siècle suivant, qu'elle devient une destination principale, en relation d'une part avec son rôle de marché d'esclaves africains à un moment où d'autres sources d'approvisionnement se tarissent, mais surtout à un moment où la réorganisation des réseaux et des routes de navigation redonne à Tripoli une place centrale d'escale sur une des routes majeures du commerce méditerranéen, à la jonction des bassins oriental et occidental de la Méditerranée. 


\section{ANNEXE}

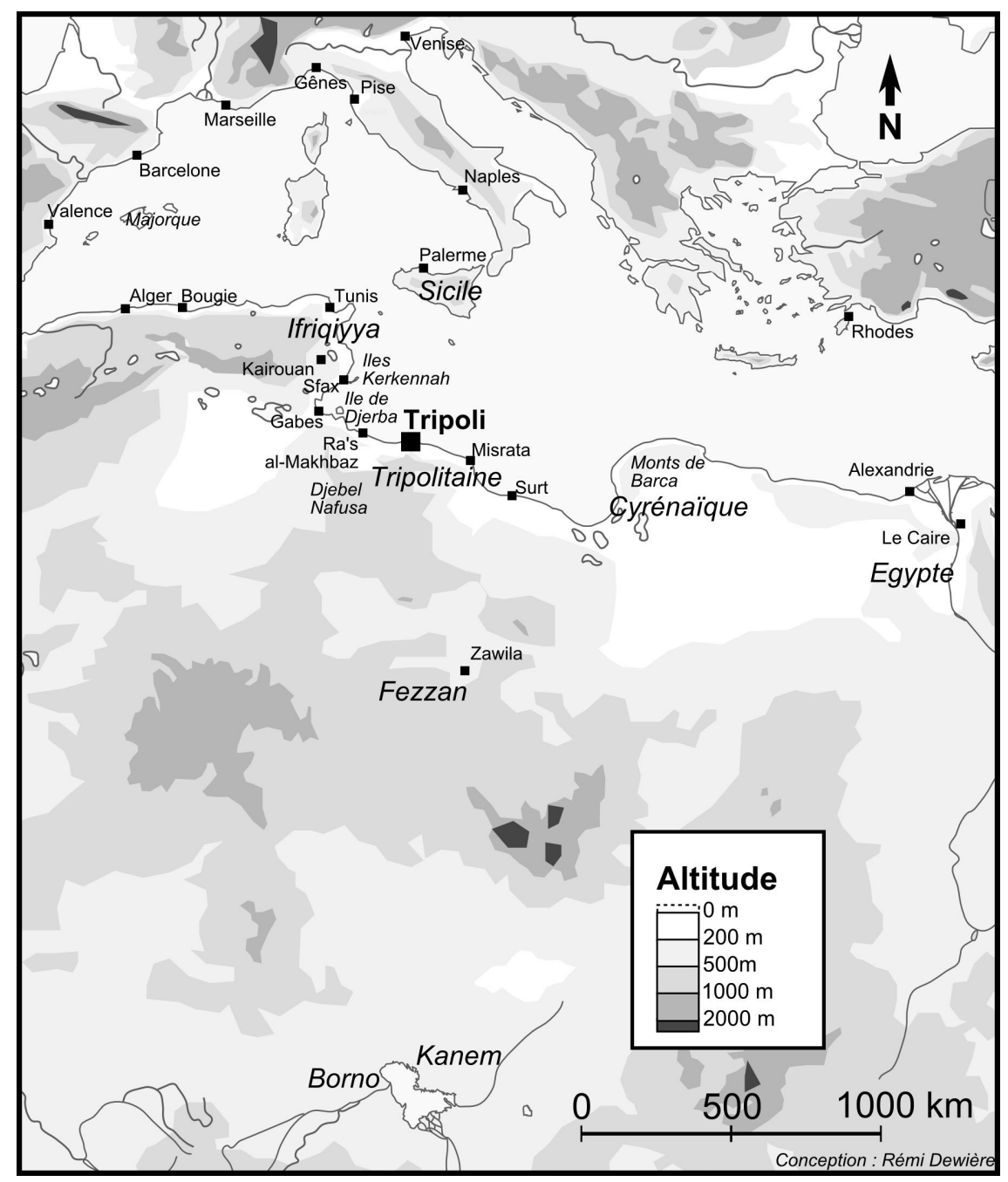

Tripoli à la fin du Moyen Âge (C Remi Dewıère 